\title{
Antibiotic resistance situation in Dhaka, Bangladesh: a review
}

\author{
M. Rashedur Rahman ${ }^{1,2 *}$, Fargana Islam ${ }^{1}$, M. Ashikur Rahman ${ }^{1}$, Shammi Binte Bashar ${ }^{1}$, \\ Rakibul Hasan Rifat ${ }^{1}$, Bidhan Chandro Saha ${ }^{1}$, M. Shah Paran ${ }^{1}$
}

${ }^{1}$ Department of Pharmacy, Primeaisa University, Banani, Dhaka, Bangladesh
${ }^{2}$ Department of Pharmacy, Jahangirnagar University, Dhaka, Bangladesh

Received: 15 March 2021

Revised: 13 April 2021

Accepted: 14 April 2021

*Correspondence:

M. Rashedur Rahman,

Email: rashib.ju@gmail.com

Copyright: $@$ the author(s), publisher and licensee Medip Academy. This is an open-access article distributed under the terms of the Creative Commons Attribution Non-Commercial License, which permits unrestricted non-commercial use, distribution, and reproduction in any medium, provided the original work is properly cited.

\begin{abstract}
Antibiotic resistance is global trouble and in the megacities, it is causing more rapidly due to the misuse and overuse of antibiotics. This systematic evaluation used to be carried out to summarize the contemporary day kingdom of affairs of antibiotic resistance in Dhaka, to emerge as aware of gaps in close observation, and to prink tips primarily based on honesty and surely on the findings. Google scholar, PubMed, and Bangladeshi journals online have been searched for the use of applicable key phrases to select articles connected to antibiotic resistance in Dhaka, Bangladesh published between 2004 to 2020. The resistance of a bacterium to a given drug was once added as the median resistance and interquartile fluctuate. Forty-one articles have been blanketed in this systematic review. Antimicrobial susceptibility trying out used to be once as quickly as carried out via disk diffusion approach in $97.56 \%$ of studies, at the equal time as the clinical and laboratory standards institute suggestions had been accompanied in $92.68 \%$. Data concerning the susceptibility attempting out method and furnish of sickness (hospital/community) had been absent in $12.19 \%, 10.52 \%$, and $90.24 \%$ of the research, respectively. An excessive prevalence of resistance used to be detected in most examined pathogens, and many of the normal first-line pills have been the most importantly ineffective. Resistance to carbapenems was once low in most cases. An excessive incidence of resistance to most antibiotics used to be detected, alongside necessary gaps in surveillance and facts gaps in the methodological data of the show up to be up.
\end{abstract}

Keywords: Antibiotic resistance, Dhaka, Pathogens, Ineffective, Antibiotic

\section{INTRODUCTION}

Antibiotics are indeed considered drugs that saved a lot of lives. At the same time, antibiotic was argued as a medication which is only targeted to certain microbes that causes disease incident. ${ }^{1}$

Antibiotic resistance (ABR) is a worldwide health problem because of both the use of antibiotics and in terms of their applications not permanently severe in the taint of monitoring practices, nowadays epidemiological distinguishes in various countries. The medical community suspects that the battle in opposition to the infectious diseases are had obtained, unfortunately, the progressive rate of antimicrobial resistance (AMR) produced to a great extent that faith was elusive in the last 30 years. $^{2,3}$ The option to start antibiotic resistance is attractive for several reasons where one of the most important factors is resistance genes, to inappropriate use of antibiotics. ${ }^{3,4}$ As a result, AMR increases some factors such as health care cost, morbidity, and fatality not only in developing countries but also in developed countries. ${ }^{5}$ According to the report of $\mathrm{WHO}$, it is estimated that approximately $45 \%$ of death are responsible in both 
South Asia and Africa due to multidrug resistance. Bangladesh is a developing country in South Asia where Dhaka is the very fastest-growing megacities in the world where the rate of antibiotic resistance is increasing day by day. There is a clear argument that antibiotic physician in Bangladesh prescribes antibiotics to patients based on guesswork. ${ }^{4,7,8}$

Current reports estimated that the rate of death will increase 10 million in 2050 and also in world economic output will be lost in case of actual attempts are not built to take on this threat. Current review highlights the percentage of data about antibiotic resistance scenarios in Dhaka that are found by the different published articles in journals. The ultimate goal is to ensure a recommendation for the upcoming workers and also provide direction to the policymakers tend towards with the viewpoint that optimum plan to minimize the rate of antibiotic resistance. ${ }^{9}$

Protocols to conduct current review article were developed to specify what is the scenario of ABR in Bangladesh, exactly in Dhaka. Current review will meet various research questions such as the emergence of ABR in Dhaka. Multiple search operations have been conducted between 2004 to 2020 to identify ABR in Dhaka-related articles. Searches have been conducted in Google scholar, Pubmed, BioMed, and Banglajol. In this review, various pathogens taken from the article report have been included which are meeting the (WHO) global priority list. A systemic review procedure was followed and a combination of keywords (antibiotics, resistance, Dhaka, Bangladesh, ) in the subject area related to each other. By collecting some data from different papers and presented in the table form. Here resistance pattern of the pathogen is also recorded Bacterial resistant pattern of each bacteria has been presented through the median range, with interquartile range. Data analysis was prepared by using Microsoft excel 2016.

Total 41 articles have been added to current review, out of 167. Each of these papers was published between 2004 and 2020. A total study has been conducted centering on Dhaka, the capital of Bangladesh. Different susceptibility testing procedures have been used, among them the disc diffusion method used in $97.56 \%$ (40/41) of the studies. Most of the data $92.68 \%(38 / 41)$ has been interpreted based on the guidelines of CLSI (clinical and laboratory standard institute) for antibacterial susceptibility testing. Out of 41 studies, $8(19.51 \%)$ samples were collected from UTI. Moreover, culture collected from gastroenteritis and bloodstream infection was resolved in $17.07 \%(7 / 41)$ of the studies. The summary of these studies is depicted in (Table 1). MR and IQR of 9 out of 14 selected pathogens have been calculated (Table 2). In the case of some pathogens, it was not possible to find out the IQR because their data is very low. At least three data are required to find the IQR.
Table 1: Summary of studies.

\begin{tabular}{|c|c|c|}
\hline Publication year & $\mathbf{N}$ & $\%$ \\
\hline $2004-2010$ & 7 & 17.07 \\
\hline $2011-2014$ & 11 & 26.82 \\
\hline $2015-2018$ & 18 & 43.90 \\
\hline $2019-2020$ & 5 & 12.20 \\
\hline \multicolumn{3}{|l|}{ Source of infection } \\
\hline Community-acquired & 4 & 9.75 \\
\hline Hospital-acquired & 5 & 12.19 \\
\hline Both & 4 & 9.75 \\
\hline Unknown & 28 & 68.29 \\
\hline \multicolumn{3}{|l|}{ Patient type } \\
\hline Inpatient & 15 & 36.58 \\
\hline Outpatient & 4 & 9.75 \\
\hline Inpatient and outpatient & 8 & 19.51 \\
\hline Did not mention & 14 & 34.14 \\
\hline \multicolumn{3}{|c|}{ Susceptibility testing method } \\
\hline Disk diffusion & 40 & 97.56 \\
\hline Dilution & 7 & 17.07 \\
\hline E-test & 2 & 4.87 \\
\hline \multicolumn{3}{|c|}{ Susceptibility testing standard } \\
\hline CLSI & 38 & 92.68 \\
\hline Eucast & 2 & 4.87 \\
\hline Did not mention & 1 & 2.43 \\
\hline \multicolumn{3}{|l|}{ Clinical syndrome } \\
\hline Urinary tract infection & 8 & 19.51 \\
\hline Bloodstream infection & 7 & 17.07 \\
\hline Gastroenteritis & 7 & 17.07 \\
\hline Wound infection & 1 & 2.43 \\
\hline Respiratory tract infection & 1 & 2.43 \\
\hline Multiple syndromes & 16 & 39.02 \\
\hline Unavailable & 1 & 2.43 \\
\hline
\end{tabular}

E. coli is the most common bacterium of UTI. In 19 articles, E. coli has been studied to show high resistance against drugs such as ampicillin (MR 92.9, IQR 66-100), amoxicillin (MR 90, IQR 7.7-95), cefixime (MR 65.35, IQR 44.3-73.59), ciprofloxacin (MR 55.67, IQR 35.1269.5), azithromycin (MR 55.67 IQR 44.25-74.06). Klebsiella spp shows resistance to ceftazidime (MR 60, IQR 50-100), cefuroxime (MR 48.64, IQR 23.79-51), Amoxyclav (MR 48.64, IQR 15.4-58). Pseudomonas spp shows resistance to amikacin (MR 68.7, IQR 50-70), ceftazidime (MR 66.6, IQR 25-95), azithromycin (MR 50.67, IQR 37.5-63.51), ciprofloxacin (MR 50, IQR 40.87-59). Salmonella spp shows resistance to nalidixic acid (MR 52, IQR 40-84.6). Shigella spp shows resistance to ampicillin (MR 53 (30-54.09), nalidixic acid (MR 51, IQR 34-52), chloramphenicol (MR 42, IQR 30.5-45.5). Staphylococcus aureus shows resistance to penicillin (MR 68.61, IQR 62.81-76.5), erythromycin (MR 65, IQR 43.37-82.5), tetracycline (MR 59.3, IQR 35.15-67.15), cefixime (MR 59.3, IQR 58.16-66.75). Streptococcus pneumoniae shows resistance to cotrimoxazole (MR 65.6, IQR 44.5-84.02). Ciprofloxacin (MR 64.6, IQR 46.87 -82.9). Acinetobacter spp. shows 
high resistance against penicillin (MR 100, IQR 100100), cefotaxime (MR 80, IQR 67.25-82.85) azithromycin (MR 79.20, IQR 56.28-100), cefepime (MR
75, IQR 41.5-82.74). Enterococcus spp. shows resistance to ciprofloxacin (MR 66, IQR 66.6-37.84), ceftriaxone (MR 49.32, IQR 42.66-49.66).

Table 2: Calculated MR and IQR of pathogens.

\begin{tabular}{|c|c|c|c|c|c|c|c|c|c|}
\hline Drug & $\begin{array}{l}\text { Acinetob } \\
\text { acter spp } \\
\text { MR } \\
\text { (IQR) } \\
\text { Total } \\
\text { sample }\end{array}$ & $\begin{array}{l}\text { Enteroco } \\
\text { ccus spp } \\
\text { MR } \\
\text { (IQR) } \\
\text { Total } \\
\text { sample }\end{array}$ & $\begin{array}{l}\text { Escheri } \\
\text { chia } \\
\text { coli } \\
\text { MR } \\
\text { (IQR) } \\
\text { Total } \\
\text { sample }\end{array}$ & $\begin{array}{l}\text { Klebsiell } \\
\text { a spp } \\
\text { MR } \\
\text { (IQR) } \\
\text { Total } \\
\text { sample }\end{array}$ & $\begin{array}{l}\text { Pseudom } \\
\text { onas spp } \\
\text { MR } \\
\text { (IQR) } \\
\text { Total } \\
\text { sample }\end{array}$ & $\begin{array}{l}\text { Salmon } \\
\text { ella spp } \\
\text { MR } \\
\text { (IQR) } \\
\text { Total } \\
\text { sample }\end{array}$ & $\begin{array}{l}\text { Shigella } \\
\text { spp MR } \\
\text { (IQR) } \\
\text { Total } \\
\text { sample }\end{array}$ & $\begin{array}{l}\text { Staphyloc } \\
\text { occus } \\
\text { aureus } \\
\text { MR } \\
\text { (IQR) } \\
\text { Total } \\
\text { sample }\end{array}$ & $\begin{array}{l}\text { Strepto } \\
\text { coccus } \\
\text { pneum } \\
\text { oniae } \\
\text { MR } \\
\text { (IQR) } \\
\text { Total } \\
\text { sample }\end{array}$ \\
\hline Amikacin & $\begin{array}{l}41.65 \\
(24.30- \\
59.97) \\
200\end{array}$ & N/A & $\begin{array}{l}11.5 \\
(7.24- \\
29.9) \\
1576\end{array}$ & $\begin{array}{l}19.21 \\
(14.97- \\
39.93) \\
247\end{array}$ & $\begin{array}{l}68.7(50- \\
70) 517\end{array}$ & N/A & N/A & N/A & N/A \\
\hline Ampicillin & N/A & N/A & $\begin{array}{l}92.9 \\
(66- \\
100) \\
1291\end{array}$ & N/A & N/A & $\begin{array}{l}30(28- \\
59.75) \\
7524\end{array}$ & $\begin{array}{l}53(30- \\
54.09) \\
14348\end{array}$ & N/A & N/A \\
\hline Amoxicillin & $\begin{array}{l}100(100- \\
100) 50\end{array}$ & N/A & $\begin{array}{l}90 \\
(7.7-95) \\
208\end{array}$ & N/A & N/A & N/A & N/A & N/A & N/A \\
\hline Amoxyclav & N/A & N/A & $\begin{array}{l}48.68 \\
(39- \\
52.04) \\
1095\end{array}$ & $\begin{array}{l}48.64 \\
(15.4- \\
58) \\
206\end{array}$ & N/A & N/A & N/A & N/A & N/A \\
\hline $\begin{array}{l}\text { Azithromy- } \\
\text { cin }\end{array}$ & $\begin{array}{l}79.20 \\
(56.28- \\
100) 55\end{array}$ & N/A & $\begin{array}{l}55.67 \\
(44.25- \\
74.06) \\
1426\end{array}$ & & $\begin{array}{l}50.67 \\
(37.5- \\
63.51) 16\end{array}$ & & & $\begin{array}{l}52.05 \\
(40.91-75) \\
174\end{array}$ & N/A \\
\hline Aztreonam & N/A & N/A & N/A & N/A & N/A & N/A & N/A & N/A & N/A \\
\hline Cephalexin & N/A & N/A & N/A & N/A & N/A & N/A & N/A & N/A & N/A \\
\hline Cefepime & $\begin{array}{l}75(41.5- \\
82.74) 49 \\
\end{array}$ & N/A & N/A & N/A & N/A & N/A & N/A & N/A & N/A \\
\hline Cefixime & N/A & N/A & $\begin{array}{l}65.35 \\
(44.3- \\
73.59) \\
378\end{array}$ & N/A & N/A & N/A & N/A & $\begin{array}{l}59.3 \\
(58.16- \\
66.75) 109\end{array}$ & N/A \\
\hline Cefotaxime & $\begin{array}{l}80 \\
(67.25- \\
82.85) 50\end{array}$ & N/A & N/A & N/A & N/A & N/A & N/A & N/A & N/A \\
\hline $\begin{array}{l}\text { Ceftazid- } \\
\text { ime }\end{array}$ & $\begin{array}{l}61.22 \\
(46.3- \\
67.41) \\
157\end{array}$ & & $\begin{array}{l}50 \\
(28.77- \\
71.25) \\
956\end{array}$ & $\begin{array}{l}60(50- \\
100) \\
200\end{array}$ & $\begin{array}{l}66.6(25- \\
95) \\
311\end{array}$ & N/A & N/A & N/A & N/A \\
\hline Ceftriaxone & $\begin{array}{l}50 \\
(43.06- \\
87.55) \\
123\end{array}$ & $\begin{array}{l}49.32 \\
(42.66- \\
49.66) 20\end{array}$ & $\begin{array}{l}49.32 \\
(32.35- \\
77.30) \\
1758\end{array}$ & N/A & N/A & $\begin{array}{l}3(1.85- \\
37.48) \\
1368\end{array}$ & $\begin{array}{l}2 \\
(1-8.28) \\
862\end{array}$ & $\begin{array}{l}51.6 \\
(45.65- \\
62.8) 130\end{array}$ & N/A \\
\hline Cefuroxime & $\begin{array}{l}56(41.5- \\
71.5) 53\end{array}$ & N/A & N/A & $\begin{array}{l}48.64(2 \\
3.79- \\
51) 164\end{array}$ & $\begin{array}{l}50(48.64- \\
96) \\
61\end{array}$ & N/A & N/A & $\begin{array}{l}59.3 \\
(43.15- \\
68.78) 109\end{array}$ & N/A \\
\hline $\begin{array}{l}\text { Chloramp- } \\
\text { henicol }\end{array}$ & N/A & N/A & N/A & N/A & N/A & $\begin{array}{l}20 \\
(14.5- \\
48.07) \\
2333\end{array}$ & $\begin{array}{l}42 \\
(30.5- \\
45.5) \\
3560\end{array}$ & N/A & N/A \\
\hline
\end{tabular}




\begin{tabular}{|c|c|c|c|c|c|c|c|c|c|}
\hline Drug & $\begin{array}{l}\text { Acineto- } \\
\text { bacter } \\
\text { spp } \\
\text { MR } \\
\text { (IQR) } \\
\text { Total } \\
\text { sample }\end{array}$ & $\begin{array}{l}\text { Entero- } \\
\text { coccus } \\
\text { spp } \\
\text { MR } \\
\text { (IQR) } \\
\text { Total } \\
\text { sample }\end{array}$ & $\begin{array}{l}\text { Esch- } \\
\text { erichia } \\
\text { coli } \\
\text { MR } \\
\text { (IQR) } \\
\text { Total } \\
\text { sample }\end{array}$ & $\begin{array}{l}\text { Kleb- } \\
\text { siella } \\
\text { spp } \\
\text { MR } \\
\text { (IQR) } \\
\text { Total } \\
\text { sample }\end{array}$ & $\begin{array}{l}\text { Pseudo- } \\
\text { monas } \\
\text { spp } \\
\text { MR } \\
\text { (IQR) } \\
\text { Total } \\
\text { sample }\end{array}$ & $\begin{array}{l}\text { Salmo- } \\
\text { nella } \\
\text { spp } \\
\text { MR } \\
\text { (IQR) } \\
\text { Total } \\
\text { sample }\end{array}$ & $\begin{array}{l}\text { Shigella } \\
\text { spp MR } \\
\text { (IQR) } \\
\text { Total } \\
\text { sample }\end{array}$ & $\begin{array}{l}\text { Staphylo- } \\
\text { coccus } \\
\text { aureus } \\
\text { MR } \\
\text { (IQR) } \\
\text { Total } \\
\text { sample }\end{array}$ & $\begin{array}{l}\text { Strepto } \\
\text { coccus } \\
\text { pneum } \\
\text { oniae } \\
\text { MR } \\
\text { (IQR) } \\
\text { Total } \\
\text { sample }\end{array}$ \\
\hline $\begin{array}{l}\text { Ciproflox- } \\
\text { acin }\end{array}$ & $\begin{array}{l}58.41 \\
(37.84- \\
89.2) 139\end{array}$ & $\begin{array}{l}66(66.6- \\
37.84) 68\end{array}$ & $\begin{array}{l}56.32 \\
(35.12- \\
69.5) \\
2823 \\
\end{array}$ & $\begin{array}{l}37.83 \\
(30-49) \\
272\end{array}$ & $\begin{array}{l}50 \\
(40.87- \\
59) \\
722 \\
\end{array}$ & $\begin{array}{l}18.5 \\
(0.9- \\
38.24) \\
7359 \\
\end{array}$ & $\begin{array}{l}12 \\
(3-17) \\
14394\end{array}$ & $\begin{array}{l}58.06 \\
(36.48- \\
72.35) 197\end{array}$ & $\begin{array}{l}64.6 \\
(46.87- \\
82.9) \\
179\end{array}$ \\
\hline Cloxacillin & N/A & N/A & N/A & N/A & N/A & N/A & N/A & $\begin{array}{l}50(49.3- \\
53.7) 126 \\
\end{array}$ & N/A \\
\hline Colistin & N/A & N/A & N/A & N/A & N/A & N/A & N/A & N/A & N/A \\
\hline $\begin{array}{l}\text { Co- } \\
\text { trimoxazole }\end{array}$ & $\begin{array}{l}40 \\
(24-75) \\
82\end{array}$ & N/A & $\begin{array}{l}48.6 \\
(34.02- \\
65) \\
2823 \\
\end{array}$ & $\begin{array}{l}33.3 \\
(30.33- \\
45.55) \\
237 \\
\end{array}$ & $\begin{array}{l}1.9 \\
(0-50) \\
478\end{array}$ & $\begin{array}{l}20 \\
(13.63- \\
32) \\
7477 \\
\end{array}$ & N/A & $\begin{array}{l}43.2 \\
(29.1- \\
50.3) 126\end{array}$ & $\begin{array}{l}65.6 \\
(44.5- \\
84.02) \\
179 \\
\end{array}$ \\
\hline $\begin{array}{l}\text { Doxycy- } \\
\text { cline }\end{array}$ & N/A & N/A & $\begin{array}{l}44 \\
(28.2-72) \\
1176\end{array}$ & N/A & N/A & N/A & N/A & N/A & N/A \\
\hline $\begin{array}{l}\text { Erythro- } \\
\text { mycin }\end{array}$ & N/A & N/A & N/A & N/A & N/A & N/A & N/A & $\begin{array}{l}65(43.37- \\
82.5) 34\end{array}$ & N/A \\
\hline Gentamicin & $\begin{array}{l}53.3 \\
(26.99- \\
85.8) 146\end{array}$ & $\begin{array}{l}25.65 \\
(15.5- \\
37.47) 62\end{array}$ & N/A & $\begin{array}{l}17 \\
(14.97- \\
39.93) \\
312\end{array}$ & $\begin{array}{l}50 \\
(48.64- \\
96) \\
732\end{array}$ & N/A & $\begin{array}{l}4(2.1- \\
37.71) \\
642\end{array}$ & $\begin{array}{l}51.07 \\
(72.63- \\
35.11) 176\end{array}$ & N/A \\
\hline Imipenem & $\begin{array}{l}37.45 \\
(22.97- \\
54.45) 92\end{array}$ & N/A & $\begin{array}{l}7.14 \\
(0.55- \\
93.15) \\
1639\end{array}$ & $\begin{array}{l}21.65 \\
(2.5- \\
45.82) \\
217\end{array}$ & $\begin{array}{l}50(7.95- \\
91.05) \\
708\end{array}$ & N/A & N/A & $\begin{array}{l}10(7.7- \\
52.2) 119\end{array}$ & N/A \\
\hline $\begin{array}{l}\text { Levoflox- } \\
\text { acin }\end{array}$ & N/A & N/A & $\begin{array}{l}40.97 \\
(34.4- \\
41) \\
801\end{array}$ & $\begin{array}{l}45.03 \\
(23.9- \\
62.87) \\
90\end{array}$ & $\begin{array}{l}7.3(3.65- \\
8.37) \\
427\end{array}$ & N/A & N/A & N/A & N/A \\
\hline $\begin{array}{l}\text { Merop- } \\
\text { enem }\end{array}$ & N/A & N/A & $\begin{array}{l}15(7- \\
56.99) \\
884\end{array}$ & $\begin{array}{l}28.57 \\
(17- \\
33.3) \\
100\end{array}$ & N/A & N/A & N/A & N/A & N/A \\
\hline $\begin{array}{l}\text { Nalidixic- } \\
\text { acid }\end{array}$ & N/A & N/A & N/A & $\begin{array}{l}60 \\
(30.67- \\
71.5) 24\end{array}$ & N/A & $\begin{array}{l}52(40- \\
81.6) \\
7299 \\
\end{array}$ & $\begin{array}{l}51(34- \\
52) \\
14614 \\
\end{array}$ & N/A & N/A \\
\hline $\begin{array}{l}\text { Nitrofuran- } \\
\text { toin }\end{array}$ & N/A & N/A & $\begin{array}{l}15(10- \\
47) \\
996\end{array}$ & $\begin{array}{l}33.3 \\
(17-43) \\
147\end{array}$ & $\begin{array}{l}25.67 \\
(1.01- \\
62.5) \\
59\end{array}$ & N/A & N/A & N/A & N/A \\
\hline Oxacillin & N/A & N/A & N/A & N/A & N/A & N/A & N/A & N/A & N/A \\
\hline Penicillin & N/A & N/A & N/A & N/A & N/A & N/A & N/A & $\begin{array}{l}68.61 \\
(62.81- \\
76.5) 121 \\
\end{array}$ & N/A \\
\hline $\begin{array}{l}\text { Tetracy- } \\
\text { cline }\end{array}$ & N/A & N/A & $\begin{array}{l}51.45 \\
(37.75- \\
61.92) \\
1199 \\
\end{array}$ & N/A & N/A & N/A & N/A & $\begin{array}{l}59.3 \\
(35.15- \\
67.15) 110\end{array}$ & N/A \\
\hline $\begin{array}{l}\text { Vanco- } \\
\text { mycin }\end{array}$ & N/A & N/A & N/A & N/A & N/A & N/A & N/A & $\begin{array}{l}35(32.35- \\
55.45) 119\end{array}$ & N/A \\
\hline
\end{tabular}


The frequent use of first-line antibiotics towards UTI used to be dissatisfying. A comparable state of affairs was discovered in Africa by contemporary analyses. $E$. coli bacteria are much more resistant against amoxicillin and amoxiclav. ${ }^{49}$ Additionally, motels are used to choose tablets like tigecycline, nitrofurantoin, etc., if obligatory. ${ }^{50}$ In staphylococcal groups, MRSA is associated with a high risk of mortality, length of hospitalization, and high health care center charges. Vancomycin was once as quickly as determined to be a high pleasant drug in opposition to MRSA in this research. First discovered antibiotic resistance of Streptococcus pneumonia used to be negligible, for this motive it stays a preference of treatment for pneumococcal disease. However, extraordinary penalties have been observed in research carried out in Asia. Despite its incidence in many other countries. Searching in this locate out about used to be different, with totally three out of a hundred and forty isolates strains of Enterococcus spp, set down into vancomycin-resistant (MR 0\%). Moreover, a total of three lookups examined vancomycin susceptibility, and a sizeable lookup was required to be done to acquire a definitive insight.

Significant gaps in the surveillance had been added in this research. The search has been carried out in Dhaka which is the capital of Bangladesh. Gaps in the methodological information are additionally identified. In Dhaka, the region antibiotics prescribed to victims no longer normally ever worked, as germs developed antibiotic resistance due to their irrational use, the standard for susceptibility results $(26.82 \%)$, and $(90.24 \%)$ of infections in hospital and community center. These markup questions are involving with an extremely good part of their statistics and make it tough to comparisons amongst the pinnacle notch studies. Further work will be done on ABR, to manipulate its issue. However, a giant lookup wishes to be carried out on the pathogens that have been left out due to the reality of an insufficient range of studies, as referred to in the Results section. ${ }^{51}$

There is a scarcity of recent antibiotics, it is of the utmost magnitude that the modern. This can be carried out by way of the use of overall performance of way of implementing stricter insurance plan insurance policies on antibiotic use.

\section{CONCLUSION}

The effect of antibiotic resistance is much greater in Dhaka, Bangladesh. Highly unwholesome strains of microbial were detected from various hospitals in megacity Dhaka and observed. Antibiotics prescribed in Dhaka hospitals work hard. This observation also implies misuse and overuse of antibiotics. At present, antibiotic resistance is a serious alarming condition for Dhaka residents. The situation in Dhaka is worse than in other districts in Bangladesh in terms of misuse. So, further exploration is very important. Although the current resistance data is not very available, this study has identified resistant bacterial pathogens in Dhaka. Their susceptibility pattern has also been highlighted. Hopefully, this study will help health professionals to make a decision about which antibiotic therapy to use. Moreover, it has been said that the fourth generation of cephalosphorin, amoxicillin, tetracycline has become resistant in Dhaka, according to prescription analysis. So, it will help the researchers for the next improvement.

Funding: No funding sources

Conflict of interest: None declared

Ethical approval: Not required

\section{REFERENCES}

1. Aminov RI. A brief history of the antibiotic era: lessons learned and challenges for the future. Front Microbiol. 2010;1:134.

2. Fauci A. Infectious diseases: considerations for the 21st century. Clin Infect Dis. 2001;32:675-85.

3. Antimicrobial resistance: global report on surveillance. Geneva, 2014. Available at: https://www.who.int/antimicrobial-resistance/ publications/surveillancereport/en/. Accessed on 20 January 2021.

4. O’Neill J. Tackling drug-resistant infections globally: final report and recommendations. Available at: https://amr-review.org/sites/default/ files/160518_Final\%20paper_with\%20cover.pdf. Accessed on 20 January 2021.

5. Gulen TA, Guner R, Celikbilek N, Keske S, Tasyaran M. Clinical importance and cost of bacteremia caused by nosocomial multi drug resistant Acinetobacter baumannii. Int J Infect Dis. 2015;38:32-5.

6. Faiz MA, Rahman MR. Rational antimicrobial use. J Chittagong Med Coll Assoc. 2004;15(1):1-3.

7. Global action plan on antimicrobial resistance. Available at; https://www.who.int/antimicrobialresistance/global-action-plan/en/. Accessed on 20 January 2021.

8. Global priority list of antibiotic-resistance bacteria to guide research, discovery, and development of new antibiotics. Available at: https://www.who.int/medicines/publications/WHOPPL-Short_Summary_25Feb-ET_NM_WHO.pdf. Accessed on 20 January 2021.

9. Ahmed I, Rabbi MB, Sultana S. Antibiotic resistance in Bangladesh: A systematic review. Int J Infect Dis. 2019;80:54-61.

10. Khan A, Huq S, Malek M, Hossain M, Talukder K, Faruque A, et al. Shigella serotypes among hospitalized patients in urban Bangladesh and their antimicrobial resistance. Epidemiol Infect. 2004;132(4):773-7.

11. Nahar S, Mukhopadhyay AK, Khan R, Ahmad MM, Datta S, Chattopadhyay S, et al. Antimicrobial susceptibility of Helicobacter pylori strains isolated in Bangladesh. J Clin Microbiol. 2004;42(10):48568. 
12. Brooks WA, Hossain A, Goswami D, Nahar K, Alam K, Ahmed N, et al. Bacteremic typhoid fever in children in an urban slum, Bangladesh. Emerg Infect Dis. 2005;11(2):326-9.

13. Mahbubur R, Shoma S, Rashid H, El Arifeen S, Baqui AH, Siddique A, et al. Increasing spectrum in antimicrobial resistance of Shigella isolates in Bangladesh: resistance to azithromycin and ceftriaxone and decreased susceptibility to ciprofloxacin. J Health Popul Nutr. 2007;25(2):158.

14. Shahriar M, Hossain M, Kabir S. A survey on antimicrobial sensitivity pattern of different antibiotics on clinical isolates of Escherichia coli collected from Dhaka City, Bangladesh. J Appl Sci Environ Manage. 2010;14(3):54-9.

15. Monjur F, Rizwan F, Asaduzzaman M, Nasrin N, Ghosh N, Apu A, et al. Antibiotic sensitivity pattern of causative organisms of neonatal septicemia in an urban hospital of Bangladesh. Indian $\mathrm{J}$ Med Sci. 2010;64(6):265.

16. Shill MC, Huda NH, Moain FB, Karmakar UK. Prevalence of uropathogens in diabetic patients and their corresponding resistance pattern: results of a survey conducted at diagnostic centers in Dhaka, Bangladesh. Oman Med J. 2010;25(4):282-5.

17. Khan SA, Feroz F, Noor R. Study of extendedspectrum $\beta$-lactamase-producing bacteria from urinary tract infections in Bangladesh. Tzu Chi Med J. 2013;25(1):39-42.

18. Dutta S, Hassan MR, Rahman F, Jilani MSA, Noor R. Study of antimicrobial susceptibility of clinically significant microorganisms isolated from selected areas of Dhaka, Bangladesh. Bangladesh J Med Sci. 2013;12(1):34.

19. Ud-Din AI, Wahid SU, Latif HA, Shahnaij M, Akter M, Azmi IJ, et al. Changing trends in the prevalence of Shigella species: emergence of multi-drug resistant Shigella sonnei biotype $\mathrm{g}$ in Bangladesh. PLoS One. 2013;8(12)e82601.

20. Afroz H, Hossain MM, Fakruddin M. A 6-year retrospective study of bloodstream Salmonella infection and antibiotic susceptibility of Salmonella enterica serovar Typhi and Paratyphi in a tertiary care hospital in Dhaka, Bangladesh. Tzu Chi Med J. 2014;26(2):73-8.

21. Akram F, Pietroni MA, Bardhan PK, Bibi S, Chisti MJ. Prevalence, clinical features, and outcome of pseudomonas bacteremia in under-five diarrheal children in Bangladesh. Microbiol. 2014;5(8):67-9.

22. Akter S, Shamsuzzaman S, Jahan F. Community acquired bacterial pneumonia: aetiology, laboratory detection and antibiotic susceptibility pattern. Malays J Pathol. 2014;36(2):97-103.

23. Chiou C-S, Lauderdale T-L, Phung DC, Watanabe $\mathrm{H}$, Kuo J-C, Wang P-J, et al. Antimicrobial resistance in Salmonella enterica serovar Typhi isolates from Bangladesh, Indonesia, Taiwan, and Vietnam. Antimicrob Agents Chemother. 2014; 58(11):6501-7.
24. Hossain MD, Ahsan S, Kabir MS. Antibiotic resistance patterns of uropathogens isolated from catheterized and noncatheterized patients in Dhaka, Bangladesh. Tzu Chi Med J. 2014;26(3):127-31.

25. Islam QT, Siddiqui MMR, Raz F, Asrafuzzaman M, Amin MR. Patterns of antimicrobial resistance among intensive care unit patients of a private medical college hospital in Dhaka. Bangladesh $\mathbf{J}$ Med. 2014;25(2):47-51.

26. Khanam F, Sayeed MA, Choudhury FK, Sheikh A, Ahmed D, Goswami D, et al. Typhoid fever in young children in Bangladesh: clinical findings, antibiotic susceptibility pattern and immune responses. PLoS Negl Trop Dis. 2015;9(4):e0003619.

27. Islam TAB, Shamsuzzaman S. Prevalence and antimicrobial susceptibility pattern of methicillinresistant, vancomycin-resistant, and Panton-valentine leukocidin positive Staphylococcus aureus in a tertiary care hospital Dhaka, Bangladesh. Tzu Chi Med J. 2015;27(1):10-4.

28. Aftab H, Miftahussurur M, Subsomwong P, Ahmed F, Khan AA, Yamaoka Y. Helicobacter pylori antibiotic susceptibility patterns in Bangladesh: emerging levofloxacin resistance. $\mathrm{J}$ Infect Dev Countries. 2016;10(03):245-53.

29. Ullah B, Ahmed S, Shahariar M, Yesmine S. Current trend of antibiotic resistance in lower respiratory tract infections (LRTIs): an experience in a teaching hospital in Bangladesh. Bangladesh Pharm J. 2016; 19(1):85-91.

30. Ahsan AA, Fatema K, Barai L, Faruq MO, Ahmed F, Saha DK, et al. Prevalence and antimicrobial resistance pattern of blood isolates in patients of septicemia in ICU: single centre observation. Bangladesh Crit Care J. 2016;4(2):100-4

31. Akter T, Hossain MJ, Khan MS, Sultana H, Fatema $\mathrm{K}$, Al Sanjee S, et al. Isolation, identification and antimicrobial susceptibility pattern analysis of Escherichia coli isolated from clinical samples of Bangladesh. Asian J Biomed Pharm Sci. 2016;6(54): 13.

32. Jobayer M, Afroz Z, Nahar SS, Begum A, Begum SA, Shamsuzzaman S. Antimicrobial susceptibility pattern of extended-spectrum beta-lactamases producing organisms isolated in a tertiary care hospital, Bangladesh. Int J Appl Basic Med Res. 2017;7(3):189.

33. Nazme NI, Al Amin A, Jalil F, Sultana J, Fatema NN. Bacteriological profile of urinary tract infection in children of a tertiary care hospital. Bangladesh J Child Health. 2017;41(2):77-83.

34. Roy S, Ahmed MU, Uddin BMM, Ratan ZA, Rajawat M, Mehta V, et al. Evaluation of antibiotic susceptibility in wound infections: a pilot study from Bangladesh. F1000 Res. 2017;6:2103.

35. Suchi SE, Shamsuzzaman S, Uddin BMM, Yusuf MA. Detection of virulence factors and antimicrobial resistance in enterococci isolated from urinary tract infection. Bangladesh J Infect Dis. 2017;4(2):30-4. 
36. Yasmeen BN, Islam S, Islam S, Uddin MM, Jahan R. Prevalence of urinary tract infection, its causative agents and antibiotic sensitivity pattern: a study in Northern International Medical College Hospital, Dhaka. North Int Med Coll J. 2015;7(1):105-9.

37. Mia MF, Mia MAA, Saha D, Khan AA, Chakraborty A. Antibiotic resistance pattern and stewardship programme in critical care settings. Bangla Med Res Counc Bull. 2017;43:131-7.

38. Ahmed D, Nahid MA, Sami AB, Halim F, Akter N, Sadique $\mathrm{T}$, et al. Bacterial etiology of bloodstream infections and antimicrobial resistance in Dhaka, Bangladesh, 2005-2014. Antimicrob Resist Infect Control. 2017;6(1):2.

39. Bello-López JM, Cabrero-Martínez OA, IbáñezCervantes G, et al. Horizontal gene transfer and its association with antibiotic resistance in the genus Aeromonas spp. Microorganisms. 2019;7(9):363.

40. Mamuye Y. Antibiotic resistance patterns of common gram-negative uropathogens in St. Paul's hospital millennium medical college. Ethiop J Health Sci. 2016;26(2):93-100.

41. Hoque R, Ahmed SM, Naher N. Tackling antimicrobial resistance in Bangladesh: A scoping review of policy and practice in human, animal and environment sectors. PLoS One. 2020;15(1): e0227947.

42. Shazzadul Islam, Yasif KS, Monjur-Al-Hossainand ABM. Study on the pattern of antibiotic use including the resistance episodes in Bangladesh. Dhaka Univ J Pharm Sci. 2019;18(2):135-43.

43. Rahman SR, Ahmed MF, Begum A. Occurrence of urinary tract infection in adolescent and adult women of shanty town in Dhaka City, Bangladesh. Ethiop J Health Sci. 2014;24(2):145-52.

44. Suchi SE, Shamsuzzaman S, Uddin BMM, Yusuf MA. Detection of virulence factors and antimicrobial resistance in enterococci isolated from urinary tract infection. Bangladesh J Infect Dis. 2017;4(2):30-4.

45. Haque R, Akter ML, Salam MA. Prevalence and susceptibility of uropathogens: a recent report from a teaching hospital in Bangladesh. BMC Res Notes. 2015;8(1):416.

46. Monjur F, Rizwan F, Asaduzzaman M, Nasrin N, Ghosh N, Apu A, et al. Antibiotic sensitivity pattern of causative organisms of neonatal septicemia in an urban hospital of Bangladesh. Indian J Med Sci. 2010;64(6):265.

47. Akter S, Ferdousi RS, Siddiqua M. Bacterial isolates and antimicrobial susceptibility in children with acute diarrhea at Ibn Sina Medical College, Bangladesh. Indian J Med Sci. 2017;8(2):80-6.

48. Begum YA, Talukder K, Azmi IJ, Shahnaij M, Sheikh A, Sharmin S, et al. Resistance pattern and molecular characterization of enterotoxigenic Escherichia coli (ETEC) strains isolated in Bangladesh. PLoS One. 2016;11(7):e0157415.

49. Tadesse BT, Ashley EA, Ongarello S, Havumaki J, Wijegoonewardena M, González IJ, et al. Antimicrobial resistance in Africa: a systematic review. BMC Infect Dis. 2017;17(1):616.

50. Garau J. Other antimicrobials of interest in the era of extended-spectrum beta-lactamases: fosfomycin, nitrofurantoin and tigecycline. Clin Microbiol Infect. 2008;14(1):198-20.

51. Ahmed I, Rabbi MB, Sultana S. Antibiotic resistance in Bangladesh: A systematic review. Int J Infect Dis. 2019;80:54-61.

Cite this article as: Rahman MR, Islam F, Rahman MA, Bashar SB, Rifat RH, Saha BC, et al. Antibiotic resistance situation in Dhaka, Bangladesh: a review. Int J Basic Clin Pharmacol 2021;10:584-90. 